\title{
AlpineBends - A Benchmark for Deep Learning-Based Generalisation
}

\author{
Azelle Courtial $^{\mathrm{a}, *}$, Guillaume Touya ${ }^{\mathrm{a}}$, Xiang Zhang ${ }^{\mathrm{b}}$ \\ ${ }^{a}$ LASTIG, Univ Gustave Eiffel, ENSG, IGN, F-94160, Saint Mandé, France, azelle.courtial@ign.fr, guillaume.touya@ign.fr \\ ${ }^{b}$ School of Geospatial Engineering and Science, Sun Yat-Sen University, Guangzhou 510275, China, zhangx795@mail.sysu.edu.cn \\ * Corresponding author
}

Keywords: image, roads, generalisation, evaluation

\begin{abstract}
:
Raster-based map generalization is nowadays anecdotal, as most generalization operations are performed using vector data. Vectors describe the shape of each object in the map using a set of coordinates; thus, the object delimitation is directly accessible, and the topology and distance-based relations are easy to compute. On the contrary, rasters represent a map as an image, a grid of pixel covers the target area, and each pixel is characterised by a value. This representation does not explicitly model the boundary/shape of geographic objects and the relations between them.
\end{abstract}

However, the emergence of the image-based deep learning techniques has shown an ability to process images of geographic information. The question of their adaptation for map generalization is a trendy subject: road (Courtial et al. 2020), building (Feng et al. 2019) and coastline (Du et al. 2021) generalization have been explored in recent years. Common methods for evaluating these techniques seems to be necessary for the comparison and development of this field.

Here we propose to create a benchmark for comparing raster-based methods for mountain road generalization. This dataset is a set of images produced by rasterization and division into small images of vector data produced by IGN (the French national map agency). The creation process is illustrated in Figure 1. The input dataset represents the road lines that appear at the $1: 250 \mathrm{k}$ scale, but with a $1: 25 \mathrm{k}$ scale level of detail. The target data is composed of images covering the same geographic area but containing roads generalized for the 1:250k scale. This vector dataset is produced at IGN using a combination of automatic or semi-automatic vector-based generalization algorithms (Mustière, 1998) and manual correction and updates. The creation of this input dataset thus required a selection process. We manually matched the roads from both scales to know which ones appeared at both scales. Then, the study area is split into images using the following regular square grid: each tile represents $2.5 \mathrm{~km}$ square and there is a recovering of 40 per cent between to neighbouring tiles. and roads are rasterized with a width that corresponds to their importance.

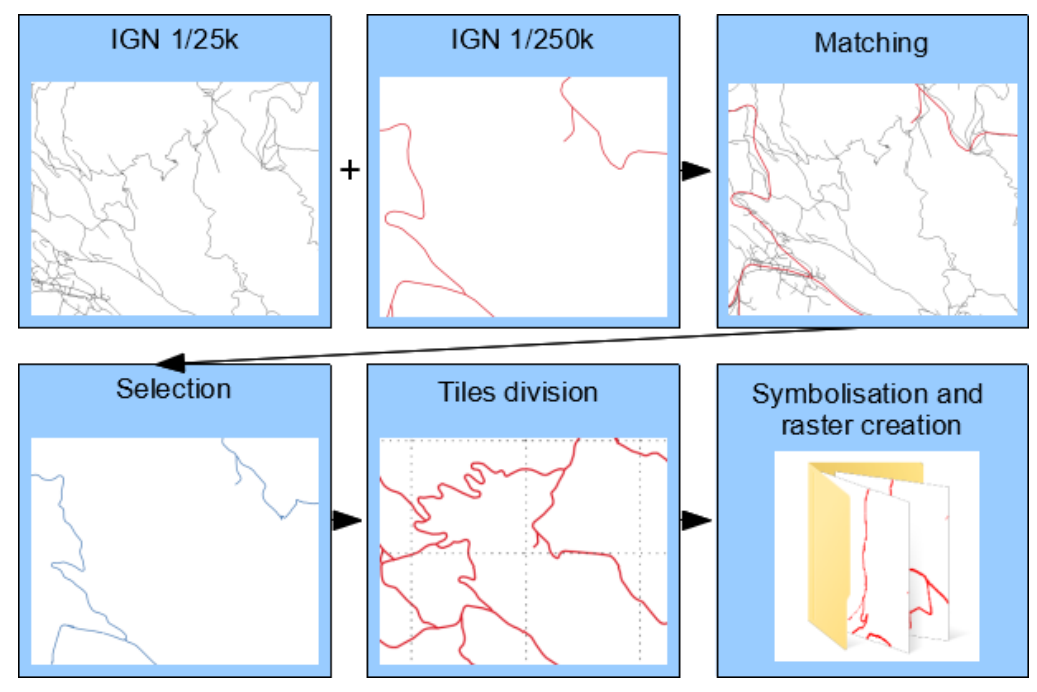

Figure 1: Tile creation process. 
The tile set represents a study area, in the north of the alps, located in Figure 2. As this dataset should be used to train and then evaluate deep learning models, the examples in this dataset should all show a good quality generalisation. In the same time, we want enough images to train the models with a subset and then evaluate them with the remaining images. A revision of the dataset might be required to guarantee both the quality and the quantity of images.

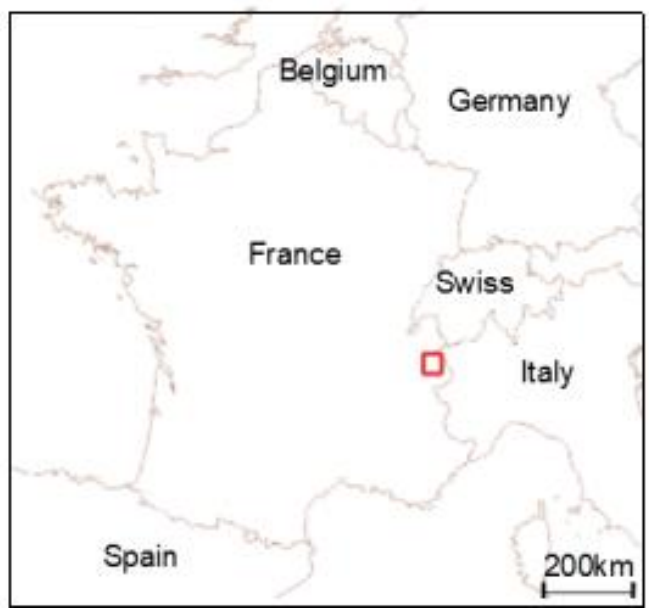

Figure 2: Area covered by the dataset.

As an evaluation, we propose to use the intersection-over-union between reference images of generalised roads and the images produced by the deep learning model tested on the benchmark (Figure 3). This evaluation permits to estimate the position accuracy of the produced roads, as well as the similarity of the output shapes.
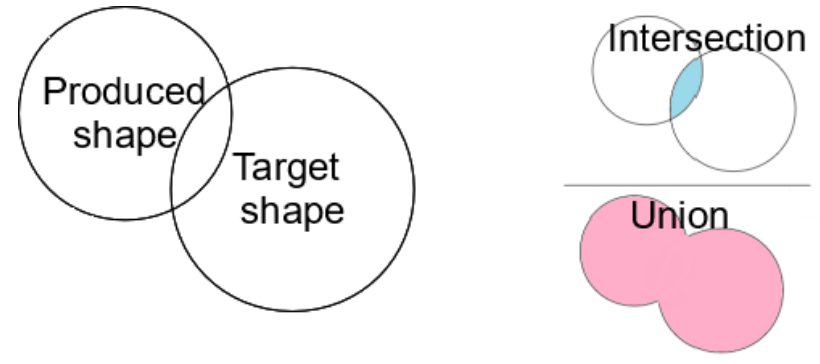

Figure 3: Evaluation measure, intersection over-union.

However, some limits remain in this evaluation: the position is not the most important factor in map generalization as displacement are tolerated and, some errors like network disconnections are important in the generalization task but may not affect this measure. A network continuity preservation measure could be appropriate to complete this benchmark.

\section{Dataset:}

Our dataset is available here: $10.5281 /$ zenodo.5257686

\section{References:}

Courtial, A.; El Ayedi, A.; Touya, G.; Zhang, X. Exploring the Potential of Deep Learning Segmentation for Mountain Roads Generalisation. ISPRS Int. J. Geo-Inf. 2020, 9, 338. https://doi.org/10.3390/ijgi9050338

Du J.; Wu F.; Xing R.; Li J.; Gong X. An Automated Approach to Coastline Simplification for Maritime Structures with Collapse Operation, Marine Geodesy, 2021, 44:3, 157-195, DOI: 10.1080/01490419.2021.1887014

Feng, Y.; Thiemann, F.; Sester, M. Learning Cartographic Building Generalization with Deep Convolutional Neural Networks. ISPRS Int. J. Geo-Inf. 2019, 8, 258. https://doi.org/10.3390/ijgi8060258

Mustière, S. GALBE: Adaptive Generalisation. The need for an Adaptive Process for Automated Generalisation, an Example on Roads. Geographic Information Systems PlaNet; 1998. 